\title{
Distributed Detection and Isolation of Sensor Faults in HVAC Systems*
}

\author{
Vasso Reppa, Panayiotis Papadopoulos, Marios M. Polycarpou, and Christos G. Panayiotou
}

\begin{abstract}
This paper presents the design of a methodology for distributed detection and isolation of sensor faults in heating, ventilating and air-conditioning (HVAC) systems. The proposed sensor fault detection and isolation (SFDI) method is developed in a distributed framework with the HVAC system modeled as a set of interconnected, nonlinear subsystems. For each of the interconnected subsystems, we design a model-based SFDI module that uses input and sensor output data of its underlying subsystems, as well as sensor output data of its neighboring, interconnected subsystems. The distributed sensor fault detection is conducted in each SFDI module, using robust analytical redundancy relations, formulated by estimationbased residuals and adaptive thresholds. The distributed sensor fault isolation is carried out by applying a diagnostic reasoningbased decision logic, taking into account multiple sensor faults. Simulation results are used for illustrating the effectiveness of the proposed methodology applied to a two-zone HVAC system.
\end{abstract}

\section{INTRODUCTION}

Recent technological advancements in home automation have contributed to the design of smart buildings. A Smart Building is a building that incorporates computer technology to autonomously govern and adapt the building environment in order to increase energy efficiency and cost effectiveness, improve comfort, productivity and safety, and increase system robustness and reliability [1], [2]. One of the essential components of a smart building is the Heating, Ventilation and Air Conditioning (HVAC) system, which is responsible for providing a high quality and healthy environment for the building's inhabitants.

The HVAC is a highly complex, nonlinear system, comprised of a set of interconnected subsystems, including the heating and cooling plant (boilers, chillers, dehumidifier), the ventilation system (Volume Air Volume (VAV) terminal units, Air Handling Unit (AHU)), and one or more zones served by the terminal units of the ventilation system. Each subsystem consists of several components, such as sensors (e.g. temperature, humidity), electrical and mechanical actuators (e.g. coils, dampers, valves) and controllers. Over time, it is inevitable that one or more HVAC components (sensors, actuators) will fail. Such failures are generally difficult to detect, especially in large buildings, and can result in significant energy waste and unfavorable living conditions. According to the Electrical and Mechanical Services Department of Hong Kong, $15 \%$ to $30 \%$ of the energy waste in

\footnotetext{
* This research has been funded by the European Commission 7th Framework Program, under grant INSFO-ICT- 270428 (i-Sense).

The authors are with the KIOS Research Center for Intelligent Systems and Networks, Department of Electrical and Computer Engineering, University of Cyprus, Nicosia, 1678, Cyprus \{vreppa, ppapad01\}@ucy.ac.cy, \{mpolycar, christosp\}@ucy.ac.cy
}

commercial buildings is due to the performance degradation, improper control strategy and faults in HVAC systems [3]. On the other hand, a successful fault detection and isolation (FDI) mechanism [4], [5], which may be one of the main functionalities of a smart building, can save $10 \%$ to $40 \%$ of the HVAC energy consumption [6].

During the last two decades, various methodologies have been developed for detecting and isolating faults in HVAC systems [7]-[10]. A large number of these methodologies have focused on the detection and isolation of faults in actuators and the plant of the HVAC system. However, the detection and isolation of sensor faults has become a challenging problem, since a large number of sensors is used for monitoring and control of the energy and the living conditions of large-scale smart buildings; in a single zone (e.g. room, corridor), there may be a temperature sensor, humidity sensor, $\mathrm{CO}_{2}$ sensor, infrared occupancy sensor, while in the electromechanical part of HVAC there may be sensors for measuring supply/return/mixed air temperature, supply/return air flow sensor, differential pressure sensor, return air humidity and many more. Faults in these sensors may have different effects; a fault in the zone temperature sensor (stuck at a high temperature) can cause the continuous operation of the chiller, leading to both discomfort and increased energy consumption; or a fault in the zone $\mathrm{CO}_{2}$ sensor can give the wrong signal to the controller for adjusting the air flow of the zone, leading to improper ventilation and unfavorable working conditions.

HVAC sensor faults may also affect the functionality of supervision schemes [11], leading to wrong decisions and disorientation of remedial actions. For example, evacuation plans in case of contaminant release in a building are usually designed in combination with emergency control strategies for the HVAC system, which are activated based on the measurements of contaminant and occupancy sensors [12]; e.g. aiming at making a zone to be a safe "haven" in case of contamination, the exhaust damper in the zone may be activated for directing the contaminant to another zone, where there are no people, according to the measurements of the occupancy sensor. However, a faulty contaminant sensor may indicate low or zero levels of contamination in the zone, leading to the non-activation of the exhaust damper, while the contaminated zone may be indicated as safe. Or, the contaminant may be directed by the exhaust damper to a zone, which is indicated as safe, although it is occupied, due to a faulty occupancy sensor (stuck at zero). Moreover, multiple HVAC sensor faults in large-scale, smart buildings are likely to occur and it is more difficult to isolate due to the combinatorial complexity of their effects. 
Sensor fault detection and isolation (SFDI) methods for HVAC systems can be classified into data-driven and modelbased methods. Data-driven SFDI methods are the most commonly used for SFDI in HVAC systems, since they can be easily developed using a black-box model, without the requirement of understanding the system's model [13][18]. However, these methods need a plethora of data collected under both healthy and faulty conditions (data under faulty conditions are necessary for fault isolation), implying increased cost due to the utilization of redundant sensors beyond the ones required for proper system operation [19]. On the other hand, model-based methods require additional modeling effort, since a HVAC model with physical significance has to be developed using a priori knowledge of system process [20]-[23]. Nevertheless, the model-based SFDI methods are designed based on the data acquired by the sensors that are usually installed for control purposes [24]-[27]. Furthermore, due to the inherent complexity of the HVAC system, most SFDI approaches focus on the diagnosis of faults in one of HVAC subsystems, e.g. chiller, AHU, VAV, considering each subsystem as isolated [16], [17], [24]. However, since the HVAC system is a set of interconnected subsystems, a sensor fault that occurred in one subsystem may propagate to other neighboring subsystems though with a delay and can appear as a "new" fault.

The objective of this work is the design of a robust, model-based method for detecting and isolating multiple sensor faults affecting HVAC systems. Taking into account the nonlinear HVAC model developed in [23], [28], which consists of two zones (rooms), a cooling coil and a chiller tank, we develop a distributed SFDI methodology based on the spatial distribution of the HVAC system, i.e. modeling the HVAC system as a set of three interconnected nonlinear systems (two zones and the electromechanical part). For each nonlinear subsystem, we design a dedicated SFDI module, which is responsible for detecting and isolating the presence of sensor faults in a distributed manned. In order for each SFDI module to do so, it uses the input and output measurements of its underlying subsystem, as well as the sensor measurements or reference signals of the neighboring subsystems. In the case that there are more than one sensors monitoring and controlling a subsystem, dedicated sensor fault (SF) detectors are designed for each sensor. The SF decision logic, which is implemented in each SFDI module, relies on checking whether certain analytical redundancy relations (ARRs) are satisfied. The ARRs are formulated using estimation-based residuals and adaptive thresholds, taking into account modeling uncertainties and measurement noise. The distributed isolation of multiple faulty sensors in the HVAC system is carried out using a diagnostic reasoning-based decision logic applied to a sensor fault signature matrix.

The contributions of this research work include the development of a distributed architecture for SFDI in HVAC systems, leading to the reduction of the computational requirements compared to a centralized approach, which requires that all measurements are conveyed to a single position.
Also, the distributed architecture enhances the reliability of the SFDI method with respect to security threats. Moreover, the proposed SFDI technique is designed to be robust with respect to modeling uncertainties and measurement noise, without causing false alarms that are not only annoying to the occupants but also deceptive in emergency situations. Finally, the proposed SFDI method is designed to tackle the problem of multiple sensor faults. Multiple sensor faults have great impact on the operation of HVAC, since they may increase dramatically the HVAC energy consumption and create a very unpleasant environment for the occupants, while they can increase the probability of loss of human life in safety-critical situations.

This paper is organized as follows. The HVAC description and the problem formulation are described in Section II. The architecture of the proposed distributed SFDI methodology for nonlinear interconnected subsystems is presented in III. Simulation results of the application of the proposed SFDI architecture to the two-zone HVAC system are provided in Section IV, followed by some concluding remarks in Section $\mathrm{V}$.

\section{HVAC DESCRIPTION AND PROBLEM FORMULATION}

In this work, we consider the HVAC system presented in [23], [28], which consists of two zones that correspond to two test cells of a laboratory-scale plant, and the electromechanical part. The basic components of the electromechanical part of the HVAC, shown in Fig. 1 are the cooling coil, the chiller and the chilled water tank, the fan, the supply and return ducts and the variable air volume (VAV) boxes. The cooling coil is connected to the chiller through the chiller water tank, which determines the water that is inserted to the cooling coil. Control inputs to the HVAC system are the air flow rate to each of the two test cells (controlled through the fan and the VAV boxes) and the chilled water mass flow rate (controlled by a 3-way valve), while the control objectives are the setpoint tracking of the temperature in each test cell and the temperature in the chilled water tank. The humidity and indoor air quality are not controlled.

In order to design the controllers for achieving the aforementioned control objectives, each test cell, the cooling coil and the chiller water tank have been modeled based on the fundamental mass and energy conservation equations under the following assumptions: i) the air temperature and velocity have uniform behavior throughout a zone, ii) the transient and spatial effects are neglected at the components which exchange air, iii) at the exterior and interior surface of the zones, supply/return ducts, etc., the heat transfer is modeled using constant heat transfer coefficients, and iv) the water temperature is constant across the cross section of the tubes.

The dynamic equations of the two-zone HVAC system are 


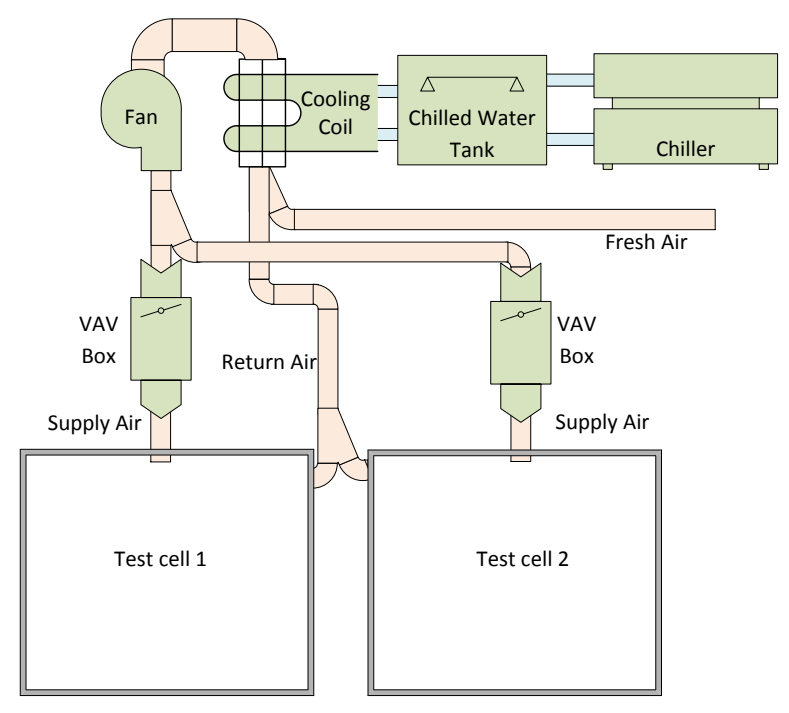

Fig. 1. Schematic of a two-zone HVAC system.

described by

$$
\begin{aligned}
M_{t c_{1}} C_{v} \frac{d T_{t c_{1}}(t)}{d t}= & \rho_{a} C_{p a}\left(T_{a o}(t)-T_{t c_{1}}(t)\right) Q_{a_{1}}(t) \\
& +U_{t c_{1}} A_{t c_{1}}\left(T_{a m b}-T_{t c_{1}}(t)\right)+\widetilde{T}_{t c_{1}}(t),(1) \\
M_{t c_{2}} C_{v} \frac{d T_{t c_{2}}(t)}{d t}= & \rho_{a} C_{p a}\left(T_{a o}(t)-T_{t c_{2}}(t)\right) Q_{a_{2}}(t) \\
& +U_{t c_{2}} A_{t c_{2}}\left(T_{a m b}-T_{t c_{2}}(t)\right)+\widetilde{T}_{t c_{2}}(t),(2) \\
M_{c c} C_{v} \frac{d T_{a o}(t)}{d t}= & \rho_{a} C_{p a}\left(Q_{a_{1}}(t)+Q_{a_{2}}(t)\right)\left(T_{t c}(t)-T_{a o}(t)\right) \\
& +U_{c c} A_{c c}\left(T_{a m b}-\left(T_{a o}(t)+T_{t c}(t)\right)\right) \\
& +Q_{w} \rho_{w} C_{p w}\left(T_{t}(t)-T_{w o}\right) \\
& +\rho_{a}\left(Q_{a_{1}}(t)+Q_{a_{2}}(t)\right)\left(h_{f g}-C_{p a}\right) w_{t c} \\
& -\rho_{a}\left(Q_{a_{1}}(t)+Q_{a_{2}}(t)\right)\left(h_{f g}-C_{p a}\right) w_{a o}, \\
= & Q_{w} \rho_{w} C_{p w}\left(T_{w o}-T_{t}(t)\right) \\
& +U_{t} A_{t}\left(T_{a m b}-T_{t}(t)\right)+\frac{15000}{V_{t} \rho_{w} C_{p w}} \chi(t) \\
M_{t} C_{v} \frac{d T_{t}(t)}{d t} &
\end{aligned}
$$

and

$$
T_{t c}(t)=\frac{T_{t c_{1}}(t)+T_{t c_{2}}(t)}{2}
$$

where $T_{t c_{i}}\left({ }^{\circ} \mathrm{C}\right)$ with $i=1,2$ is the temperature of the $i$-th test cell, $T_{a o}\left({ }^{\circ} \mathrm{C}\right)$ is the output air temperature from cooling coil and $T_{t}\left({ }^{\circ} \mathrm{C}\right)$ is the temperature of the water in the chiller storage tank. The variable $Q_{a_{i}}\left(\mathrm{~m}^{3} / \mathrm{sec}\right)$ is the volumetric flow rate of air entering into the $i$-th test cell and $\chi\left(\mathrm{m}^{3} / \mathrm{sec}\right)$ is the chilled water mass flow rate. The value $\widetilde{T}_{t c_{i}}(t)\left({ }^{\circ} \mathrm{C} / \mathrm{sec}\right)$ represents the rate of heat leakage from the ambient and internal heat gain, due to occupants and appliances from the $i$-th test cell. The constant parameters of the HVAC system are: $T_{a m b}$ is the ambient temperature, $M_{t c_{i}}(\mathrm{~kg})$ is the heat mass capacitance corresponding to the $i$-th test cell, $i=1,2$, $C_{v}(\mathrm{~J} / \mathrm{kg} \mathrm{K})$ is specific heat at constant volume, $U\left(\mathrm{~W} / \mathrm{m}^{2}\right.$ $\mathrm{K})$ is the overall heat transfer coefficient, $\rho_{a}\left(\mathrm{~kg} / \mathrm{m}^{3}\right)$ is the air density, $A\left(\mathrm{~m}^{3}\right)$ is the test cell component area, $C_{p a}(\mathrm{~J} / \mathrm{kg}$ $\mathrm{K})$ is the specific heat of air at constant pressure, $h_{f g}(\mathrm{~J} / \mathrm{kg})$ for latent heat of water and $T_{w o}\left({ }^{\circ} \mathrm{C}\right)$ is the temperature of water, $w_{t c}, w_{a o}$ are humidity factors [23].

The HVAC system described by (1)-(4) is a fourth-order nonlinear system with state vector $x=\left[T_{t c_{1}}, T_{t c_{2}}, T_{a o}, T_{t}\right]^{\top}$ and input vector $u=\left[Q_{a_{1}}, Q_{a_{2}}, \chi\right]^{\top}$. It is noted that the inputs $u_{1}$ and $u_{2}\left(u_{i}\right.$ is the $i$-th element of $\left.u\right)$ are generated by two feedback linearization controllers and $u_{3}$ is generated by a gain controller, using sensor measurements and some desired reference signals. The two-zone HVAC system can be regarded as a set of three interconnected, nonlinear subsystems that are the two test cells and the electromechanical part (cooling coil and chiller water tank).

Let us denote the $I$-th subsystem of HVAC as $\Sigma^{(I)}, I=$ $1,2,3$, characterized by the state, input and interconnection vector $x^{(I)}, u^{(I)}, z^{(I)}$, respectively. It is noted that the state and input vector $x^{(I)}, u^{(I)}$ contain elements of $x$ and $u$, respectively, which characterize only to $\Sigma^{(I)}$, while the interconnection vector $z^{(I)}$ contains the shared states between the $I$-th subsystem and its neighboring subsystems. The first and second subsystem, which correspond to the first and second test cell given in (1) and (2), respectively, are represented as:

$$
\begin{aligned}
\Sigma^{(I)}: \dot{x}^{(I)}(t)= & A^{(I)} x^{(I)}(t)+\gamma^{(I)}\left(x^{(I)}(t), u^{(I)}(t)\right) \\
& +h^{(I)}\left(z^{(I)}(t), u^{(I)}(t)\right)+\eta^{(I)}(t), I=1,2
\end{aligned}
$$

where $x^{(I)}(t)=T_{t c_{I}}(t), u^{(I)}(t)=Q_{a_{I}}(t)$ and $z^{(I)}(t)=T_{a o}(t)$, $A^{(I)}=-\frac{U_{t c_{I}} A_{t c_{I}}}{M_{t c_{I}} C_{v}}, \eta^{(I)}(t)=\frac{1}{M_{t c_{I}} C_{v}} \tilde{T}_{t c_{I}}(t)$ and

$$
\begin{aligned}
\gamma^{(I)}\left(x^{(I)}, u^{(I)}\right) & =-\frac{\rho_{a} C_{p a}}{M_{t c_{I}} C_{v}} x^{(I)}(t) u^{(I)}+\frac{U_{t c_{I}} A_{t c_{I}}}{M_{t c_{I}} C_{v}} T_{a m b} \\
h^{(I)}\left(z^{(I)}, u^{(I)}\right) & =\frac{\rho_{a} C_{p a}}{M_{t c_{I}} C_{v}} z^{(I)} u^{(I)}
\end{aligned}
$$

It is noted that $h^{(I)}$ represents the effects of the interconnection between $\Sigma^{(I)}$ and $\Sigma^{(3)}$ on $\Sigma^{(I)}$, where the subsystem $\Sigma^{(3)}$ correspond to the cooling coil along with the chiller water tank given in (3) and (4), represented as:

$$
\begin{aligned}
\Sigma^{(3)}: \dot{x}^{(3)}(t)= & A^{(3)} x^{(3)}(t)+\gamma^{(3)}\left(u^{(3)}(t)\right) \\
& +h^{(3)}\left(x^{(3)}(t), z^{(3)}(t), u_{z}^{(3)}(t)\right),
\end{aligned}
$$

where $x^{(3)}(t)=\left[x_{1}^{(3)}(t), x_{2}^{(3)}(t)\right]^{\top}=\left[T_{a o}(t), T_{t}(t)\right]^{\top}$, $z^{(3)}(t)=\left[z_{1}^{(3)}(t), z_{2}^{(3)}(t)\right]^{\top}=\left[T_{t c_{1}}(t), T_{t c_{2}}(t)\right]^{\top}$ and $u_{z}^{(3)}(t)=\left[u^{(1)}(t), u^{(2)}(t)\right]^{\top}$,

$$
\begin{aligned}
A^{(3)}= & {\left[\begin{array}{cc}
-\frac{U_{c c} A_{c c}}{M_{c c} C_{v}} & \frac{Q_{w} \rho_{w} C_{p w}}{M_{c c} C_{v}} \\
0 & -\frac{Q_{w} \rho_{w} C_{p w}+U_{t} A_{t}}{V_{t} \rho_{w} C_{p w}}
\end{array}\right], } \\
\gamma^{(3)}\left(u^{(3)}\right)= & {\left[\begin{array}{c}
\frac{U_{c c} A_{c c}}{M_{c c} C_{v}} T_{a m b}-\frac{Q_{w} \rho_{w} C_{p w}}{M_{c c} C_{v}} T_{w o} \\
\frac{U_{t} A_{t}}{V_{t} \rho_{w} C_{p w}} T_{a m b}+\frac{Q_{w} \rho_{w} C_{p w}}{V_{t} \rho_{w} C_{p w}} T_{w o}
\end{array}\right] } \\
& +\left[\begin{array}{c}
0 \\
\frac{15000}{V_{t} \rho_{w} C_{p w}}
\end{array}\right] u^{(3),}
\end{aligned}
$$




$$
h^{(3)}\left(x^{(3)}, z^{(3)}, u_{z}^{(3)}\right)=\left[\begin{array}{c}
h_{1}^{(3)}\left(x^{(3)}, z^{(3)}, u_{z}^{(3)}\right) \\
0
\end{array}\right]
$$

with

$$
\begin{aligned}
h_{1}^{(3)}\left(x^{(3)}, z^{(3)}, u_{z}^{(3)}\right)= & \left(\frac{\rho_{a} C_{p a}}{M_{c c} C_{v}}\left[\begin{array}{l}
1 \\
1
\end{array}\right]^{\top} u_{z}^{(3)}\right. \\
& \left.-\frac{U_{c c} A_{c c}}{M_{c c} C_{v}}\right)\left[\begin{array}{c}
\frac{1}{2} \\
\frac{1}{2}
\end{array}\right]^{\top} z^{(3)} \\
& +\frac{\rho_{a}}{M_{c c} C_{v}}\left(\left(h_{f g}-C_{p a}\right)\left(w_{t c}-w_{a o}\right)\right. \\
& \left.-C_{p a}\left[\begin{array}{l}
1 \\
0
\end{array}\right]^{\top} x^{(3)}\right)\left[\begin{array}{l}
1 \\
1
\end{array}\right]^{\top} u_{z}^{(3)}
\end{aligned}
$$

The nonlinear subsystem $\Sigma^{(I)}, I=1,2,3$ is monitored and/or controlled by $m_{I}$ sensors, which are assumed to constitute a sensor set $\mathcal{S}^{(I)}=\left\{\mathcal{S}^{(I)}\{1\}, \ldots, \mathcal{S}^{(I)}\left\{m_{I}\right\}\right\}$, where $\mathcal{S}^{(I)}\{j\}, j \in\left\{1, \ldots, m_{I}\right\}$, denotes the $j$-th sensor. Particularly, the sensor sets $\mathcal{S}^{(1)}$ and $S^{(2)}$ contain a single sensor measuring the test cell temperature $T_{t c_{i}}$, i.e. are singleton sets $\left(m_{1}=m_{2}=1\right)$, while $\mathcal{S}^{(3)}$ contains two sensors $\left(m_{3}=2\right)$ such that $\mathcal{S}^{(3)}\{1\}$ measures the output air temperature of the cooling coil, $T_{a o}$, and $\mathcal{S}^{(3)}\{2\}$ measures the temperature of water in the chiller tank. The sensor set $\mathcal{S}^{(I)}$ is described by

$$
\mathcal{S}^{(I)}: y^{(I)}(t)=x^{(I)}(t)+d^{(I)}(t)+f^{(I)}(t),
$$

where $y^{(I)} \in \mathbb{R}^{m_{I}}$ is the sensor output vector, $d^{(I)} \in \mathbb{R}^{m_{I}}$ denotes the noise vector corrupting the measurements of sensors in $\mathcal{S}^{(I)}$ and $f^{(I)} \in \mathbb{R}^{m_{I}}$ represents the possible sensor fault vector. The $j$-th sensor, $j \in\left\{1, \ldots, m_{I}\right\}$, is characterized by

$$
\mathcal{S}^{(I)}\{j\}: y_{j}^{(I)}(t)=x_{j}^{(I)}(t)+d_{j}^{(I)}(t)+f_{j}^{(I)}(t),
$$

where $f_{j}^{(I)}$ represents the change in the output $y_{j}^{(I)}$ due to a single fault in the $j$-th sensor and can be expressed as

$$
f_{j}^{(I)}(t)=\beta_{j}^{(I)}\left(t-T_{f_{j}}^{(I)}\right) \phi_{j}^{(I)}\left(t-T_{f_{j}}^{(I)}\right),
$$

where $\beta_{j}^{(I)}(t)$ is the time profile and $\phi_{j}^{(I)}(t)$ is the (unknown) function of the sensor fault that occurs at the (unknown) time instant $T_{f_{j}}^{(I)}$. The time profile of the fault is modeled as $\beta_{j}^{(I)}(t)=0$ if $t<0$, and $\beta_{j}^{(I)}(t)=1-e^{-\kappa_{j}^{(I)} t}$, if $t \geq 0$, where $\kappa_{j}^{(I)}>0$ denotes the (unknown) evolution rate of the fault. If the occurrence of a sensor fault is abrupt, $\kappa_{j}^{(I)} \rightarrow \infty$.

The objective of this work is to design a distributed methodology for detecting and isolating multiple sensor faults that may occur in the sensor sets $\mathcal{S}^{(I)}, I=1,2,3$, used for monitoring and control of the HVAC system, under the following assumptions:

Assumption 1: The state vector $x^{(I)}$ and input vector $u^{(I)}$ generated by a feedback controller, remain bounded for all $I=1,2,3$ before and after the occurrence of multiple sensor faults, i.e. there are compact regions of stability $\mathcal{U}_{I} \subset \mathbb{R}^{\ell_{I}}$, $\mathcal{X}_{I} \subset \mathbb{R}^{n_{I}}$ for $u^{(I)}(t)$ and $x^{(I)}(t)$, respectively, such that $\left(x^{(I)}(t), u^{(I)}(t)\right) \in \mathcal{X}_{I} \times \mathcal{U}_{I}$, for all $t>0$.
Assumption 2: The unknown modeling uncertainty of $\Sigma^{(I)}$, $\eta^{(I)}(t), I=1,2$ and the noise corrupting the measurements of each sensor in $\mathcal{S}^{(I)}$, are unknown but uniformly bounded:

$$
\begin{aligned}
& \left|\eta^{(I)}(t)\right| \leq \bar{\eta}^{(I)}, I=1,2 \\
& \left|d_{i}^{(I)}(t)\right| \leq \bar{d}_{i}^{(I)}, \quad i \in\left\{1, \ldots, m_{I}\right\}, I=1,2,3
\end{aligned}
$$

where $\bar{\eta}^{(I)}, \bar{d}_{i}^{(I)}$ are known constant bounds.

Assumption 1 entails that the feedback controller, which generates the input $u^{(I)}$, can retain the boundedness of the state variables even in the presence of multiple sensor faults. This assumption is required due to the fact that, in this work, we deal exclusively with the fault detection and isolation problem, not the fault accommodation issue. Assumption 2 is needed to ensure robustness of the sensor fault detection and isolation scheme with respect to modeling errors and measurement noise.

\section{DISTRIBUTED SENSOR FAULT DETECTION AND ISOLATION}

This section provides the design details of the distributed architecture for detection and isolation of sensor faults in the HVAC system described in Section II. The first step is to design a sensor fault detection and isolation (SFDI) module dedicated to each of the interconnected subsystems described by (6) and (9), as shown in Fig. 2 [29], [30]. Each SFDI module has access to the input and output data of the underlying subsystem, i.e. $u^{(I)}, y^{(I)}$, and may use some sensor data of its neighboring subsystems and prior information related to the reference signals of its neighboring subsystems $r^{(I)}(t), I=1,2,3$, which are transmitted by its neighboring modules [31]. For control purposes of the twozone HVAC system, $r^{(1)}$ and $r^{(2)}$ are the reference signals for the test cell temperature and $r^{(3)}$ is the reference signal for the chiller water temperature.

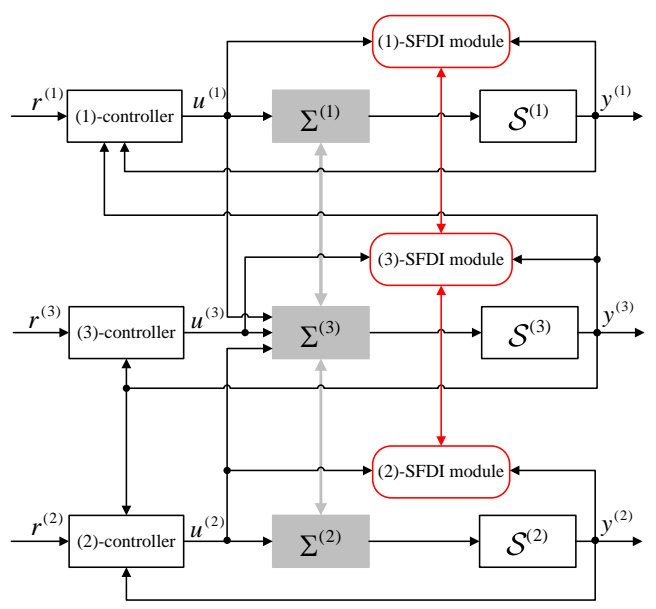

Fig. 2. Distributed sensor fault detection and isolation architecture for interconnected nonlinear systems.

The primary role of the $(I)$-SFDI module is to detect the presence of sensor faults in its underlying sensor set 
$\mathcal{S}^{(I)}, I=1,2,3$. Due to the fact that the sensor fault (SF) detection in the $(I)$-SFDI module is carried out using sensor measurements of neighboring modules, and assuming the occurrence of multiple sensor faults in more than one sensor sets $\mathcal{S}^{(I)}$, the information (decision) generated by the three SFDI modules is combined and processed using a diagnostic reasoning logic for distributed sensor fault isolation, as shown in Fig. 3.

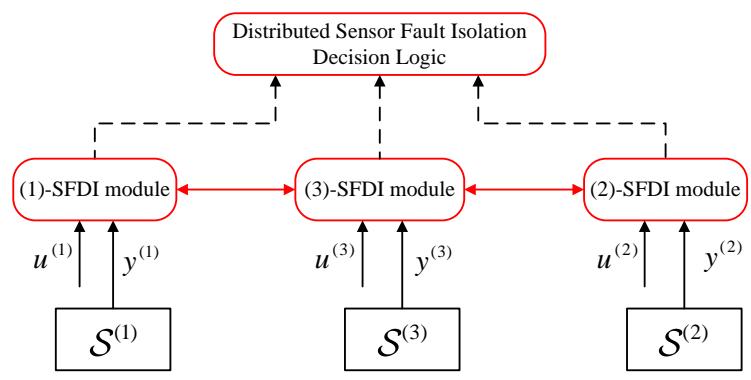

Fig. 3. Distributed sensor fault detection isolation.

Since the sensor sets $\mathcal{S}^{(1)}$ and $\mathcal{S}^{(2)}$ are singletons, the (1)and (2)-SFDI module are dedicated to the sensors $\mathcal{S}^{(1)}\{1\}$ and $\mathcal{S}^{(2)}\{1\}$. However, the sensor set $\mathcal{S}^{(3)}$ contains two sensors. Hence, under the assumption of multiple sensor fault occurrence in $\mathcal{S}^{(3)}$, we design two SF detectors that are dedicated to each of these sensors, i.e. $\mathcal{S}^{(3)}\{1\}, \mathcal{S}^{(3)}\{2\}$. The design of the SFDI modules and the distributed sensor fault detection and isolation decision logic are provided next.

\section{A. Residual generation}

The sensor fault detection decision logic that is implemented in the $(I)$-SFDI module, $I=1,2,3$, relies on the comparison of $m_{I}$ residuals to $m_{I}$ adaptive thresholds that are computed using an estimator.

The structure of the nonlinear estimator, which is implemented in the $(I)$-SFDI module, $I=1,2$ and uses the measurements of $\mathcal{S}^{(I)}$, is described by the following equation:

$$
\begin{aligned}
\dot{\hat{x}}^{(I)}(t)= & A^{(I)} \hat{x}^{(I)}(t)+\gamma^{(I)}\left(y_{1}^{(I)}(t), u^{(I)}(t)\right) \\
& +h^{(I)}\left(y_{1}^{(3)}(t), u^{(I)}(t)\right)+L^{(I)}\left(y_{1}^{(I)}(t)-\hat{x}^{(I)}(t)\right),
\end{aligned}
$$

where $\hat{x}^{(I)} \in \mathbb{R}$ is the estimation of $x^{(I)}$, with initial conditions $\hat{x}^{(I)}(0)=0, L^{(I)} \in \mathbb{R}$ is the estimator gain, chosen such that the $(I)$-estimator is stable, and $y_{1}^{(I)}, y_{1}^{(3)}$ are the output of sensors $\mathcal{S}^{(1)}\{1\}$ and $\mathcal{S}^{(2)}\{1\}$, respectively, defined in (15).

Let us define $\varepsilon_{x}^{(I)} \triangleq x^{(I)}-\hat{x}^{(I)}$ as the state estimation error for $I=1,2$; taking into account (6) and (19), it yields:

$$
\begin{aligned}
\dot{\varepsilon}_{x}^{(I)}(t)= & A_{L}^{(I)} \varepsilon_{x}^{(I)}(t)+\eta^{(I)}(t)-L^{(I)}\left(d^{(I)}(t)+f^{(I)}(t)\right) \\
& +\gamma^{(I)}\left(x^{(I)}(t), u^{(I)}(t)\right)-\gamma^{(I)}\left(y^{(I)}(t), u^{(I)}(t)\right) \\
& +h^{(I)}\left(z^{(I)}(t), u^{(I)}(t)\right)-h^{(I)}\left(y_{1}^{(3)}(t), u^{(I)}(t)\right)
\end{aligned}
$$

where $A_{L}^{(I)}=A^{(I)}-L^{(I)}$ is designed to have strictly negative eigenvalues. The residual that is generated by the $(I)$-SFDI module, $I=1,2$, is denoted as $\varepsilon_{y}^{(I)} \in \mathbb{R}$ and is described by

$$
\varepsilon_{y}^{(I)}(t)=\varepsilon_{x}^{(I)}(t)+d_{1}^{(I)}(t)+f_{1}^{(I)}(t),
$$

where $\varepsilon_{x}^{(I)}$ is the solution of (20). The analytical description of the residual $\varepsilon_{y}^{(I)}, I=1,2$ is given by the following equation:

$$
\begin{aligned}
\varepsilon_{y}^{(I)}(t)= & e^{A_{L}^{(I)} t} \varepsilon_{x}^{(I)}(0)+d_{1}^{(I)}(t)+f_{1}^{(I)}(t) \\
& +\int_{0}^{t} e^{A_{L}^{(I)}(t-\tau)}\left(\eta^{(I)}(\tau)-L^{(I)}\left(d_{1}^{(I)}(\tau)+f_{1}^{(I)}(\tau)\right)\right. \\
& +\gamma^{(I)}\left(x^{(I)}(\tau), u^{(I)}(\tau)\right)-\gamma^{(I)}\left(y^{(I)}(\tau), u^{(I)}(t)\right) \\
& \left.+h^{(I)}\left(z^{(I)}(\tau), u^{(I)}(\tau)\right)-h^{(I)}\left(y_{1}^{(3)}(\tau), u^{(I)}(\tau)\right)\right) d \tau .
\end{aligned}
$$

Based on (22), the residual $\varepsilon_{y}^{(I)}$ is affected by a fault in sensor $\mathcal{S}^{(I)}\{1\}$ and sensor $\mathcal{S}^{(3)}\{1\}$, defined in (15) for $j=1$.

The (3)-SFDI module includes two SF detectors with each detector dedicated to a sensor of the set $\mathcal{S}^{(3)}$. Let us denote the $j$-th detector, $j=1,2$ as $(3, j)$-SF detector; the estimator in the $(3,1)$-SF detector (denoted as $(3,1)$ estimator) is structured as follows:

$$
\begin{aligned}
\dot{\hat{x}}^{(3,1)}(t)= & A^{(3)} \hat{x}^{(3,1)}(t)+\gamma^{(3)}\left(u^{(3)}(t)\right) \\
& +h^{(3)}\left(y_{1}^{(3)}(t), z_{r}^{(3)}(t), u_{z}^{(3)}(t)\right) \\
& +L^{(3,1)}\left(y_{1}^{(3)}(t)-C^{(3,1)} \hat{x}^{(3,1)}(t)\right),
\end{aligned}
$$

where $\hat{x}^{(3)} \in \mathbb{R}^{2}$ is the estimation of $x^{(3)}$, with initial conditions $\hat{x}^{(3)}(0)=0, L^{(3,1)} \in \mathbb{R}^{2 \times 1}$ is the estimator gain matrix, chosen such that the $(3,1)$-estimator is stable, $C^{(3,1)}=[1,0]^{\top}$ and $z_{r}^{(3)}(t)=\left[r^{(1)}, r^{(2)}\right]^{\top}$, i.e. $z_{r}^{(3)}$ contains the a priori known reference signals used for controlling $T_{t c_{1}}$ and $T_{t c_{2}}$.

Let us define $\varepsilon_{x}^{(3,1)} \triangleq x^{(3)}-\hat{x}^{(3,1)}$; based on (9) and (23), it yields

$$
\begin{aligned}
\dot{\varepsilon}_{x}^{(3,1)}(t)= & A_{L}^{(3,1)} \varepsilon_{x}^{(3,1)}(t)-L^{(3,1)}\left(d_{1}^{(3)}(t)+f_{1}^{(3)}(t)\right) \\
& +h^{(3)}\left(x^{(3)}(t), z^{(3)}(t), u_{z}^{(3)}(t)\right) \\
& -h^{(3)}\left(y_{1}^{(3)}(t), z_{r}^{(3)}(t), u_{z}^{(3)}(t)\right),
\end{aligned}
$$

where $A_{L}^{(3,1)}=A^{(3)}-L^{(3,1)} C^{(3,1)}$ is designed to have strictly negative eigenvalues. The residual, which is generated by the $(3,1)$-SF detector, is denoted as $\varepsilon_{y}^{(3,1)}$ and is described by

$$
\varepsilon_{y}^{(3,1)}(t)=C^{(3,1)} \varepsilon_{x}^{(3,1)}(t)+d_{1}^{(3)}(t)+f_{1}^{(3)}(t),
$$

where $\varepsilon_{x}^{(3,1)}$ is the solution of (24). The analytical expression of the residual $\varepsilon_{y}^{(3,1)}$ is presented below:

$$
\begin{aligned}
\varepsilon_{y}^{(3,1)}(t)= & C^{(3,1)} e^{A_{L}^{(3,1)} t} \varepsilon_{x}^{(3,1)}(0)+d_{1}^{(3)}(t)+f_{1}^{(3)}(t) \\
& +\int_{0}^{t} C^{(3,1)} e^{A_{L}^{(3,1)}(t-\tau)}\left(-L^{(3,1)} d_{1}^{(3)}(\tau)\right. \\
& -L^{(3,1)} f_{1}^{(3)}(\tau)+h^{(3)}\left(x^{(3)}(\tau), z^{(3)}(\tau), u_{z}^{(3)}(\tau)\right) \\
& \left.-h^{(3)}\left(y_{1}^{(3)}(\tau), z_{r}^{(3)}(\tau), u_{z}^{(3)}(\tau)\right)\right) d \tau .
\end{aligned}
$$


According to (26), the residual $\varepsilon_{y}^{(3,1)}$ is affected only by a fault in sensor $\mathcal{S}^{(3)}\{1\}$, defined in (15) for $j=1$.

The estimator in the $(3,2)-\mathrm{SF}$ detector (denoted as $(3,2)$ estimator) is structured as follows:

$$
\begin{aligned}
\dot{\hat{x}}^{(3,2)}(t)= & A_{22}^{(3)} \hat{x}^{(3,2)}(t)+\gamma_{2}^{(3)}\left(u^{(3)}(t)\right) \\
& +L^{(3,2)}\left(y_{2}^{(3)}(t)-\hat{x}^{(3,2)}(t)\right)
\end{aligned}
$$

where $\hat{x}^{(3,2)} \in \mathbb{R}$ is the estimation of $x_{2}^{(3)}$, with initial conditions $\hat{x}^{(3,2)}(0)=0, L^{(3,2)} \in \mathbb{R}$ is the estimator gain chosen such that the $(3,2)$-estimator is stable, $A_{22}^{(3)}$ is the element $\{2,2\}$ of the matrix $A^{(3)}$, and $y_{2}^{(3)}$ is the output of sensor $\mathcal{S}^{(3)}\{2\}$, defined in (15).

Let us define $\varepsilon_{x}^{(3,2)} \triangleq x_{2}^{(3)}-\hat{x}^{(3,2)}$; based on (9) and (27), it yields

$$
\dot{\varepsilon}_{x}^{(3,2)}(t)=A_{L}^{(3,2)} \varepsilon_{x}^{(3,2)}(t)-L^{(3,2)}\left(d_{2}^{(3)}(t)+f_{2}^{(3)}(t)\right),
$$

where $A_{L}^{(3,2)}=A_{22}^{(3)}-L^{(3,2)}$ is designed to have strictly negative eigenvalues.. The residual, which is generated by the $(3,2)$-SF detector, is denoted as $\varepsilon_{y}^{(3,2)}$ and is expressed as:

$$
\varepsilon_{y}^{(3,2)}(t)=\varepsilon_{x}^{(3,2)}(t)+d_{2}^{(3)}(t)+f_{2}^{(3)}(t),
$$

where $\varepsilon_{x}^{(3,2)}$ is the solution of (28). The analytical expression of the residual $\varepsilon_{y}^{(3,2)}$ is

$$
\begin{aligned}
\varepsilon_{y}^{(3,2)}(t)= & e^{A_{L}^{(3,2)} t} \varepsilon_{x}^{(3,2)}(0)+d_{2}^{(3)}(t)+f_{2}^{(3)}(t) \\
& -\int_{0}^{t} e^{A_{L}^{(3,2)}(t-\tau)} L^{(3,2)}\left(d_{2}^{(3)}(\tau)+f_{2}^{(3)}(\tau)\right) d \tau .
\end{aligned}
$$

According to (30), the residual $\varepsilon_{y}^{(3,2)}$ is affected only by a fault in sensor $\mathcal{S}^{(3)}\{2\}$, defined in (15) for $j=2$.

\section{B. Computation of adaptive thresholds}

The adaptive threshold is a time-varying function that bounds a residual under healthy conditions. The objective is to make the sensor fault detection scheme robust with respect to modeling uncertainties and noise. Hence, the adaptive threshold that is implemented in the $(I)$-SFDI module, denoted as $\bar{\varepsilon}_{y}^{(I)}, I=1,2$, is computed such that $\left|\varepsilon_{y}^{(I)}(t)\right| \leq \bar{\varepsilon}_{y}^{(I)}(t)$, where $\varepsilon_{y}^{(I)}$ is the residual determined under healthy conditions. Particularly, by bounding (22), we obtain

$$
\begin{aligned}
\left|\varepsilon_{y}^{(I)}(t)\right| \leq & \left|e^{A_{L}^{(I)} t}\right|\left|\varepsilon_{x}^{(I)}(0)\right|+\left|d^{(I)}(t)\right|+ \\
& +\int_{0}^{t} e^{A_{L}^{(I)}(t-\tau)}\left(\left|\eta^{(I)}(\tau)\right|+\left|L^{(I)}\right|\left|d^{(I)}(\tau)\right|\right. \\
& +\left|\gamma^{(I)}\left(x^{(I)}(\tau), u^{(I)}(\tau)\right)-\gamma^{(I)}\left(y^{(I)}(\tau), u^{(I)}(t)\right)\right| \\
& \left.+\left|h^{(I)}\left(z^{(I)}(\tau), u^{(I)}(\tau)\right)-h^{(I)}\left(y_{1}^{(3)}(\tau), u^{(I)}(\tau)\right)\right|\right) d \tau
\end{aligned}
$$

Consider that there are: i) a known bound $\bar{x}^{(I)}$ such that $\left|x^{(I)}(0)\right| \leq \bar{x}^{(I)}$, and ii) positive constants $\rho^{(I)}, \xi^{(I)}$ such that $\left|e^{A_{L}^{(I)} t}\right| \leq \rho^{(I)} e^{-\xi^{(I)} t}$ for all $t$. Moreover, given (7), (8) and (14), (15) under healthy conditions it yields:

$$
\begin{aligned}
\left|\gamma^{(I)}\left(x^{(I)}, u^{(I)}\right)-\gamma^{(I)}\left(y^{(I)}(\tau), u^{(I)}(t)\right)\right| & \leq b_{1}\left|u^{(I)}\right| \bar{d}_{1}^{(I)}, \\
\left|h^{(I)}\left(z^{(I)}, u^{(I)}\right)-h^{(I)}\left(y_{1}^{(3)}, u^{(I)}\right)\right| & \leq b_{1}\left|u^{(I)}\right| \bar{d}_{1}^{(3)}
\end{aligned}
$$

where $b_{1}=\frac{\rho_{a} C_{p a}}{M_{t c_{I}} C_{v}}$. Taking into account Assumptions 1 and 2 , the adaptive threshold $\bar{\varepsilon}_{y}^{(I)}$ is expressed as

$$
\begin{aligned}
\bar{\varepsilon}_{y}^{(I)}(t)= & \rho^{(I)} e^{-\xi^{(I)} t} \bar{x}^{(I)}+\bar{d}_{1}^{(I)}+\int_{0}^{t} \rho^{(I)} e^{-\xi^{(I)}(t-\tau)}\left(\bar{\eta}^{(I)}\right. \\
& \left.+\left|L^{(I)}\right| \bar{d}_{1}^{(I)}+b_{1}\left(\bar{d}_{1}^{(I)}+\bar{d}_{1}^{(3)}\right)\left|u^{(I)}(\tau)\right|\right) d \tau
\end{aligned}
$$

It is noted that the adaptive threshold can be implemented using linear filtering techniques; let us denote $E^{(I)}(t)$ the integral term in (34); then

$$
\begin{aligned}
E^{(I)}(t)= & H(s)\left[\bar{\eta}^{(I)}+\left|L^{(I)}\right| \bar{d}^{(I)}\right. \\
& \left.+b_{1}\left(\bar{d}^{(I)}+\bar{d}_{1}^{(3)}\right)\left|u^{(I)}(t)\right|\right], \\
H(s)= & \frac{\rho^{(I)}}{s+\xi^{(I)}} .
\end{aligned}
$$

Following the same procedure, the adaptive threshold that is implemented in the $(3,1)-\mathrm{SF}$ detector of the (3)SFDI module, denoted as $\bar{\varepsilon}_{y}^{(3,1)}$, is computed such that $\left|\varepsilon_{y}^{(3,1)}(t)\right| \leq \bar{\varepsilon}_{y}^{(3,1)}(t)$, where $\varepsilon_{y}^{(3,1)}$ is the residual determined under healthy conditions. Taking into account Assumption 2 and that there are a known bound $\bar{x}^{(3)}$ such that $\left|x^{(3)}(0)\right| \leq \bar{x}^{(3)}$, and positive constants $\rho^{(3,1)}, \xi^{(3,1)}$ such that $\left|C^{(3,1)} e^{A_{L}^{(3,1)} t}\right| \leq \rho^{(3,1)} e^{-\xi^{(3,1)} t}$ for all $t$, the adaptive threshold is defined as

$$
\begin{aligned}
\bar{\varepsilon}_{y}^{(3,1)}(t)= & \int_{0}^{t} \rho^{(3,1)} e^{-\xi^{(3,1)}(t-\tau)}\left(\left|L^{(3,1)}\right| \bar{d}_{1}^{(3)}+\bar{h}^{(3)}(\tau)\right) d \tau \\
& +\rho^{(3,1)} e^{-\xi^{(3,1)} t} \bar{x}^{(3)}+\bar{d}_{1}^{(3)}
\end{aligned}
$$

where $\bar{h}^{(3)}(t)$ is computed such that $\left|h^{(3)}\left(x^{(3)}, z^{(3)}, u_{z}^{(3)}\right)-h^{(3)}\left(y_{1}^{(3)}, z_{r}^{(3)}, u_{z}^{(3)}\right)\right| \leq \bar{h}^{(3)}$, i.e.

$$
\begin{aligned}
\bar{h}^{(3)}(t)= & \left|\frac{\rho_{a} C_{p a}}{M_{c c} C_{v}}\left(u^{(1)}(t)+u^{(2)}(t)\right)-\frac{U_{c c} A_{c c}}{M_{c c} C_{v}}\right| \frac{1}{2}\left(\bar{z}_{1}^{(3)}+\bar{z}_{2}^{(3)}\right) \\
& +\frac{\rho_{a} C_{p a}}{M_{c c} C_{v}} d_{1}^{(3)}\left|u^{(1)}(t)+u^{(2)}(t)\right|
\end{aligned}
$$

where $\bar{z}_{j}^{(3)}, j=1,2$ is a known constant bound that is related to the tracking error, i.e. $\lim _{t \rightarrow \infty}\left|z_{j}^{(3)}(t)-z_{r_{j}}^{(I)}(t)\right|=$ $\lim _{t \rightarrow \infty}\left|T_{t c_{j}}(t)-r^{(j)}\right| \leq \bar{z}_{j}^{(I)}, \forall t>0$.

Finally, the adaptive threshold that is implemented in the $(3,2)$-SF detector of the (3)-SFDI module, denoted as $\bar{\varepsilon}_{y}^{(3,2)}$, is computed such that $\left|\varepsilon_{y}^{(3,2)}(t)\right| \leq \bar{\varepsilon}_{y}^{(3,2)}(t)$, where $\varepsilon_{y}^{(3,2)}$ is the residual determined under healthy conditions and the adaptive threshold $\bar{\varepsilon}_{y}^{(3,2)}$ is described by

$$
\begin{aligned}
\bar{\varepsilon}_{y}^{(3,2)}(t)= & \rho^{(3,2)} e^{-\xi^{(3,2)} t} \bar{x}^{(3,2)}+\bar{d}_{2}^{(3)} \\
& +\int_{0}^{t} \rho^{(3,2)} e^{-\xi^{(3,2)}(t-\tau)}\left|L^{(3,2)}\right| \bar{d}_{2}^{(3)} d \tau
\end{aligned}
$$


where $\bar{x}^{(3,2)}$ is a known bound such that $\left|x_{2}^{(3)}(0)\right| \leq \bar{x}^{(3,2)}$, and $\rho^{(3,2)}, \xi^{(3,2)}$ are positive constants such that $\left|e^{A_{L}^{(3,2)}} t\right| \leq$ $\rho^{(3,2)} e^{-\xi^{(3,2)} t}$ for all $t$.

\section{Distributed sensor fault detection and isolation decision logic}

The primary goal of the $(I)$-SFDI module $I=1,2,3$ is to detect faults in the underlying sensor set $\mathcal{S}^{(I)}$. In this work, the sensor fault detection decision logic in the $(I)$-SFDI module relies on checking whether analytical redundancy relations (ARRs) are satisfied [32]. The ARRs are formulated using the estimator-based residuals and the adaptive thresholds. It is important to note that the designed ARRs are robust with respect to modeling uncertainties and measurement noise and structured, i.e. are sensitive not to all sensor faults but to a smaller set of sensor faults.

Particularly, the SF detection decision logic of $(I)$-SFDI module, $I=1,2$ is based on the following ARR:

$$
\mathcal{E}^{(I)}: \quad\left|\varepsilon_{y}^{(I)}(t)\right|-\bar{\varepsilon}_{y}^{(I)}(t) \leq 0, I=1,2,
$$

where $\varepsilon_{y}^{(I)}$ and $\bar{\varepsilon}_{y}^{(I)}$ are defined in (22) and (34), respectively. The $\operatorname{ARR} \mathcal{E}^{(I)}$ is satisfied under healthy conditions, since the adaptive threshold $\bar{\varepsilon}_{y}^{(I)}$ bounds by design the residual $\varepsilon_{y}^{(I)}$ under healthy conditions (see Section III-B).

In the (3)-SFDI module, the SF detection decision logic is implemented in each of the SF detectors based on the following ARRs:

$$
\begin{array}{ll}
\mathcal{E}^{(3,1)}: & \left|\varepsilon_{y}^{(3,1)}(t)\right|-\bar{\varepsilon}_{y}^{(3,1)}(t) \leq 0, \\
\mathcal{E}^{(3,2)}: & \left|\varepsilon_{y}^{(3,2)}(t)\right|-\bar{\varepsilon}_{y}^{(3,2)}(t) \leq 0
\end{array}
$$

where $\varepsilon_{y}^{(3,1)}, \varepsilon_{y}^{(3,2)}$ and $\bar{\varepsilon}_{y}^{(3,1)}, \bar{\varepsilon}_{y}^{(3,2)}$ are defined in (26), (30) and (37), (39), respectively. The ARRs $\mathcal{E}^{(3,1)}$ and $\mathcal{E}^{(3,2)}$ are satisfied under healthy conditions, since the adaptive threshold are designed to bound the corresponding residuals under healthy condition.

Based on the previous analysis, the $(I)$-SFDI module detect the presence of sensor faults, if $\mathcal{E}^{(I)} I=1,2,3$ is violated. The output of the $(I)$-SFDI module, $I=1,2$ is the decision about the presence of sensor faults, which is represented by a boolean function, defined as

$$
D^{(I)}(t)=\left\{\begin{array}{ll}
0, & \text { if } t<T_{F D}^{(I)} \\
1, & \text { if } t \geq T_{F D}^{(I)}
\end{array},\right.
$$

where $T_{F D}^{(I, q)}$ is the time instant of the detection of sensor faults by the $(I)$-SFDI module, defined as:

$$
T_{F D}^{(I)}=\min \left\{t:\left|\varepsilon_{y}^{(I)}(t)\right|-\bar{\varepsilon}_{y}^{(I)}(t)>0\right\},
$$

while the decision of the $(3, j)$-SF detector in the $(3)$-SFDI module is described by

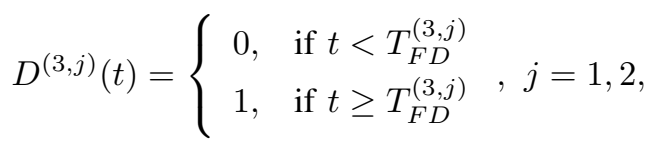

$$
T_{F D}^{(3, j)}=\min \left\{t:\left|\varepsilon_{y}^{(3, j)}(t)\right|-\bar{\varepsilon}_{y}^{(3, j)}(t)>0\right\} .
$$

The distributed sensor fault isolation decision logic is realized taking into account the the decision functions $D^{(I)}$, $I=1,2$ and $D^{(3, j)}, j=1,2$ (see Fig. 3) and the sensor fault signature matrix (SFSM). In general, a SFSM is a binary matrix, whose columns correspond to ARRs and rows to all possible combinations of a number of sensor faults. In this work, three SFSM, with the $I$-th SFSM denoted as $(I)$-SFSM $I=1,2,3$, are designed as shown in Tables I and II.

TABLE I

(I)-SFSM, $I=1,2$

\begin{tabular}{|c|c|c|c|}
\cline { 2 - 4 } \multicolumn{1}{c|}{} & $f_{1}^{(I)}$ & $f_{1}^{(3)}$ & $\left\{f_{1}^{(I)}, f_{1}^{(3)}\right\}$ \\
\hline $\mathcal{E}^{(I)}$ & 1 & 1 & 1 \\
\hline $\mathcal{E}^{(3,1)}$ & 0 & 1 & 1 \\
\hline
\end{tabular}

TABLE II

(3)-SFSM.

\begin{tabular}{|c|c|c|c|}
\cline { 2 - 4 } \multicolumn{1}{c|}{} & $f_{1}^{(3)}$ & $f_{2}^{(3)}$ & $\left\{f_{1}^{(3)}, f_{2}^{(3)}\right\}$ \\
\hline $\mathcal{E}^{(3,1)}$ & 1 & 0 & 1 \\
\hline $\mathcal{E}^{(3,2)}$ & 0 & 1 & 1 \\
\hline
\end{tabular}

The rationale behind the design of (1)- and (2)-SFSM relies on the distributed deployment of the three SFDI modules. The $(I)$-SFDI module, $I=1,2$, uses the measurements of $y_{1}^{(3)}$ for computing the residuals, adaptive thresholds and ARRs. The inference about the presence of sensor faults in $\mathcal{S}^{(I)}$ should be realized by combining the decision of the $(I)$ SFDI module and $(3,1)$-SF detector of the (3)-SFDI module. The $(I)$-SFDI module could isolate sensor faults in $\mathcal{S}^{(I)}$ in a decentralized manner, if it had not used the measurements of $y_{1}^{(3)}$, conveyed by the (3)-SFDI module. On the other hand, the (3)-SFDI module can detect faults in sensors $\mathcal{S}^{(3)}\{1\}$, $\mathcal{S}^{(3)}\{2\}$ without using measurements of sensors in $\mathcal{S}^{(1)}$ and $\mathcal{S}^{(2)}$. Hence, it can perform SF detection, irrespective of the decision generated by the (1)- and (2)-SFDI module.

Based on the previous analysis, the columns of the SFSM presented in Table I correspond the ARRs $\mathcal{E}^{(I)}$ and $\mathcal{E}^{(3,1)}$ and its rows are the combination of faults $f_{1}^{(I)}, f_{1}^{(3)}$, $I=1,2$, while the columns of the (3)-SFSM, presented in Table II, correspond to the ARRs of the $(3,1)$ - and $(3,2)$ SF detector and its rows correspond to the combination of faults in $\mathcal{S}^{(3)}\{1\}, \mathcal{S}^{(3)}\{2\}$. The columns of $(I)$-SFSM, $I=1,2,3$, represent the theoretical patterns of sensor fault combinations; i.e. the $i$-th theoretical sensor fault pattern, denoted as $M_{f c_{i}}^{(I)}, i=1,2,3$, is a binary vector, with each element been equal to 1 if at least one of the $i$-th sensor fault combination is involved in $\mathcal{E}^{(I)}$ or $\mathcal{E}^{(3,1)}$. All the theoretical sensor fault patterns of the (3)-SFSM are unique in contrast to the theoretical fault patterns of $(I)$-SFSM.

The distributed isolation of sensor faults is realized by comparing the theoretical sensor fault patterns of the three 
SFSMs to the observed patterns of sensor faults, defined as:

$$
\begin{aligned}
& p_{o}^{(I)}(t)=\left[D^{(I)}(t), D^{(3,1)}(t)\right]^{\top}, I=1,2, \\
& p_{o}^{(3)}(t)=\left[D^{(3,1)}(t), D^{(3,2)}(t)\right]^{\top},
\end{aligned}
$$

where $D^{(I)}, I=1,2$ and $D^{(3, j)}, j=1,2$ are defined in (43) and (45), respectively. If $p_{o}^{(I)}(t)=M_{f c_{i}}^{(I)}, I=$ $1,2,3$, then we can determine the sensor fault diagnosis set $\mathcal{D}_{s}^{(I)}(t)$ that contains the combination(s) of sensor faults, whose theoretical fault pattern(s) is (are) consistent to the observed pattern. When a theoretical sensor fault pattern is unique and is consistent to the observed sensor fault pattern, then the sensor fault diagnosis set contains one sensor fault combination. In the opposite case, the sensor fault diagnosis set contains more than one sensor fault combinations.

Based on the $(I)$-SFSM presented in Table I and applying the distributed sensor fault isolation decision logic, we may infer that: i) if $p_{o}^{(I)}(t)=[1,0]^{\top}$, then $\mathcal{D}_{s}^{(I)}(t)=\left\{f_{1}^{(I)}\right\}$ implying that $\mathcal{S}^{(I)}\{1\}$ is faulty, and ii) if $p_{o}^{(I)}(t)=[1,1]^{\top}$, then $\mathcal{D}_{s}^{(I)}(t)=\left\{f_{1}^{(3)},\left\{f_{1}^{(I)}, f_{1}^{(3)}\right\}\right\}$ implying that $\mathcal{S}^{(3)}\{1\}$ is faulty or both $\mathcal{S}^{(3)}\{1\}$ and $\mathcal{S}^{(I)}\{1\}$ are faulty. In any case, the sensor $\mathcal{S}^{(3)}\{1\}$ is faulty. Taking into account the (3)-SFSM presented in Table II, we may infer that: i) if $p_{o}^{(3)}(t)=[1,0]^{\top}$, then $\mathcal{D}_{s}^{(3)}(t)=\left\{f_{1}^{(3)}\right\}$ and sensor $\mathcal{S}^{(3)}\{1\}$ is faulty, ii) if $p_{o}^{(I)}(t)=[0,1]^{\top}$, then $\mathcal{D}_{s}^{(3)}(t)=\left\{f_{2}^{(3)}\right\}$ and sensor $\mathcal{S}^{(3)}\{2\}$ is faulty, and iii) if $p_{o}^{(I)}(t)=[1,1]^{\top}$, then $\mathcal{D}_{s}^{(3)}(t)=\left\{\left\{f_{1}^{(3)}, f_{2}^{(3)}\right\}\right\}$ implying that both sensors $\mathcal{S}^{(3)}\{1\}$ and $\mathcal{S}^{(3)}\{2\}$ are faulty. It is noted that all sensor fault combinations are isolable except for the combinations $\left\{f_{1}^{(3)}\right\}$ and $\left\{f_{1}^{(I)}, f_{1}^{(3)}\right\}, I=1,2$, since they have the same theoretical sensor fault patterns. However, if the effects of the interconnection between $\Sigma^{(I)}$ and $\Sigma^{(3)}$ on $\Sigma^{(I)}$ are weak, then the effects of a fault in $\Sigma^{(3)}\{1\}$ may also be weak. This fact may lead to the generation of an observed pattern $p_{o}^{(I)}(t)=[0,1]^{\top}$, based on which we may infer that only sensor fault $f_{1}^{(3)}$ has occurred and we may exclude the occurrence of $f_{1}^{(I)}$.

\section{SIMULATION RESULTS}

The objective of this section is to illustrate the effectiveness of the proposed distributed SFDI method applied to the two-zone model of the HVAC system described in Section II by simulating a number of scenarios assuming the occurrence of single sensor fault and multiple sensor faults.

The operation of the HVAC system is simulated based on equations (6)-(13). The dimensions of each test cell is $3.5 \mathrm{~m} \times 1.75 \mathrm{~m} \times 2 \mathrm{~m}$. The parameters used for the simulation of the $I$-th subsystem $I=1,2$ given in (6) and (8) are: $A^{(I)}=-0.0006, \frac{\rho_{a} C_{p a}}{M_{t c_{I}} C_{v}}=0.1144, \frac{U_{t c_{I}} A_{t c_{I}}}{M_{t c_{I}} C_{v}}=0.0006$, $T_{a m b}=35$. The parameters used for the simulation of $\Sigma^{(3)}$ described by (9)-(13) are: $\frac{U_{c c} A_{c c}}{M_{c} C_{v}}=0.02815$, $\frac{Q_{w} \rho_{w} C_{p w}}{M_{c c} C_{v}}=1.2084 \quad$ and $\quad \frac{Q_{w} \rho_{w} C_{p w} C_{t} C_{t} A_{t}}{V_{t} \rho_{w} C_{p w}}=0.0007$, $T_{w o}=5, \quad \frac{U_{c c} A_{c c}}{M_{c c} C_{v}}=0.02815, \quad \frac{Q_{w} \rho_{w} C_{p w}}{M_{c c} C_{v}}=1.2084$,
$\frac{U_{t} A_{t}}{V_{t} \rho_{w} C_{p w}}=5.4566 \times 10^{-4}, \quad \frac{Q_{w} \rho_{w} C_{p w}}{V_{t} \rho_{w} C_{p w}}=1.544 \times 10^{-5} \quad$ and $\frac{15000}{V_{t} \rho_{w} C_{p w}}=0.006$. The function $h^{(3)}$ is defined using the parameters $\frac{\rho_{a} C_{p a}}{M_{c c} C_{v}}=3.932, \quad \frac{U_{c c} A_{c c}}{M_{c c} C_{v}}=0.02815$ and $\frac{\rho_{a}}{M_{c c} C_{v}}\left(\left(h_{f g}-C_{p a}\right)\left(w_{t c}-w_{a o}\right)=0.0005\right.$.

The modeling uncertainty of $\Sigma^{(I)}, \eta^{(I)}(t)$ for $I=1,2$, is simulated as $\eta^{(I)}(t)=5 \% Y^{(I)} \sin 2 \pi f_{\eta}^{(I)}(t)$, where $Y^{(I)}$ is the steady state value of the $I$-th test cell temperature under healthy conditions, while the noise of each sensor is uniformly distributed satisfying (18) with $\bar{d}_{i}^{(I)}=5 \% Y^{(I)}$.

Following the design of the distributed SFDI method provided in Section III, we design four estimators, structured as in (19), (23) and (27) with estimator gains: $L^{(I)}=3, I=$ $1,2, L^{(3)}=[4.9712,5.1692]^{\top}$ and $L^{(3,2)}=3$. The adaptive thresholds given in (34), (37) and (39) are designed using the following parameters: $p^{(I)}=1, \xi^{(I)}=2.5, p^{(3,1)}=2.5$, $\xi^{(3,1)}=14, p^{(3,2)}=1.5$ and $\xi^{(3,2)}=3$.

Table III presents the eight sensor fault scenarios, which were simulated assuming both the occurrence of a single sensor fault and the occurrence of multiple sensor faults (the semantics of the $\{i, j\}$-element of the table being equal to 1 is that the sensor fault in the $j$-th column has been simulated in the $i$-th fault scenario). In all scenarios, we assume the occurrence of abrupt, bias sensor faults, whose magnitude and time instant of occurrence are: $\phi_{1}^{(1)}(t)=15 \% Y^{(1)}$ and $T_{f_{1}}^{(1)}=5000 \mathrm{sec}, \phi_{1}^{(2)}(t)=15 \% Y^{(2)}$ and $T_{f_{1}}^{(2)}=5100 \mathrm{sec}$, $\phi_{1}^{(3)}(t)=10 \% Y_{1}^{(3)}$ and $T_{f_{1}}^{(3)}=5200 \mathrm{sec}$, and $\phi_{2}^{(3)}(t)=$ $10 \% Y_{2}^{(3)}$ and $T_{f_{2}}^{(3)}=5300 \mathrm{sec}$. The results of the application

TABLE III

\begin{tabular}{|c|c|c|c|c|c|}
\hline & \multicolumn{4}{|c|}{ Sensor Faults } \\
\hline & & $f_{1}^{(1)}$ & $f_{1}^{(2)}$ & $f_{1}^{(3)}$ & $f_{2}^{(3)}$ \\
\hline \multirow{4}{*}{ Single-Fault Scenario } & 1 & 1 & 0 & 0 & 0 \\
\hline & 2 & 0 & 1 & 0 & 0 \\
\hline & 3 & 0 & 0 & 1 & 0 \\
\hline & 4 & 0 & 0 & 0 & 1 \\
\hline \multirow{4}{*}{ Multiple-Fault Scenario } & 1 & 1 & 1 & 0 & 0 \\
\hline & 2 & 1 & 0 & 1 & 0 \\
\hline & 3 & 0 & 0 & 1 & 1 \\
\hline & 4 & 1 & 0 & 1 & 1 \\
\hline
\end{tabular}

SIMULATED SINGLE- AND MULTIPLE-FAULT SCENARIOS

of the distributed SFDI method to the HVAC system are illustrated in Fig. 4 and 5. Particularly, Fig. 4 presents the distributed sensor fault isolation decision in the case that only a single sensor fault has occurred, while Fig. 5 presents the distributed sensor fault isolation decision in the case of multiple sensor faults. Each subfigure (Fig. 4(a)-4(d) and 5(a)-5(d)) includes four plots, showing the time evolution of the boolean decision function of the (1)-SFDI module, the (2)-SFDI module, the $(3,1)$-SF detector and the $(3,2)$ SF detector. The four plots of each subfigure can be used to determine the three observed patterns, given in (47) and (48), for each fault scenario. Hence, comparing these plots 

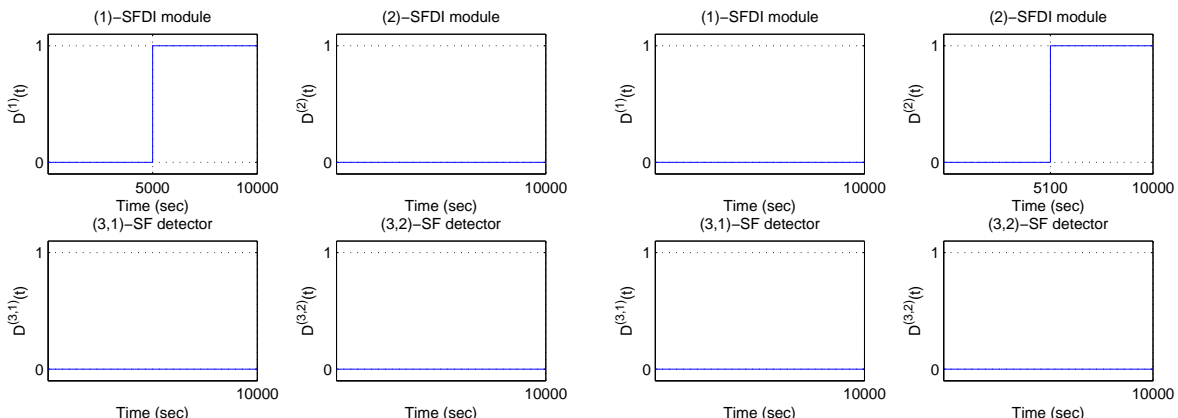

(a) Scenario 1

(b) Scenario 2
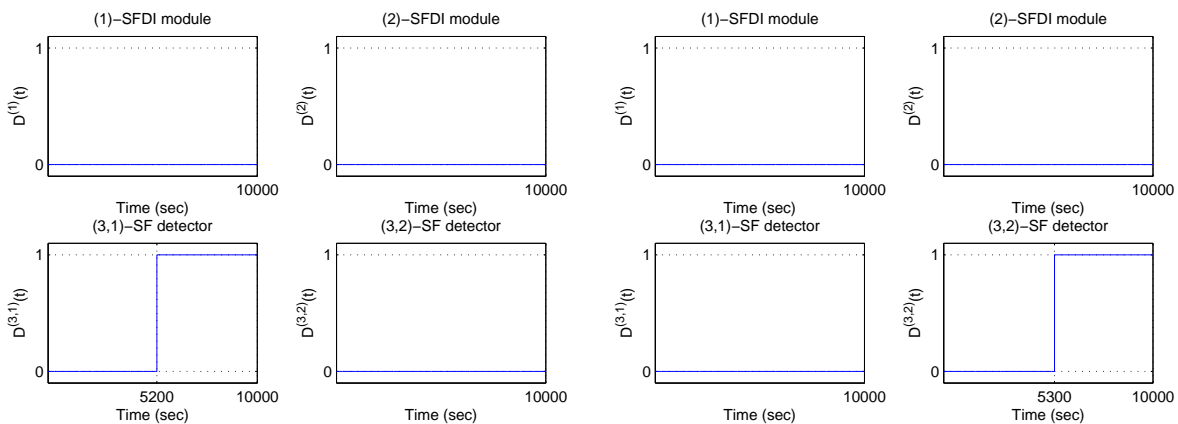

(c) Scenario 3
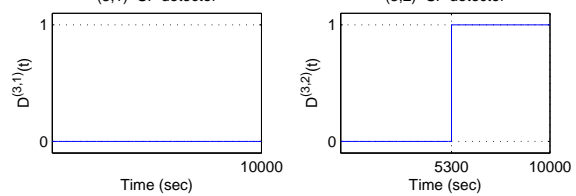

(d) Scenario 4

Fig. 4. Distributed sensor fault detection and isolation decision in the case of a single sensor fault
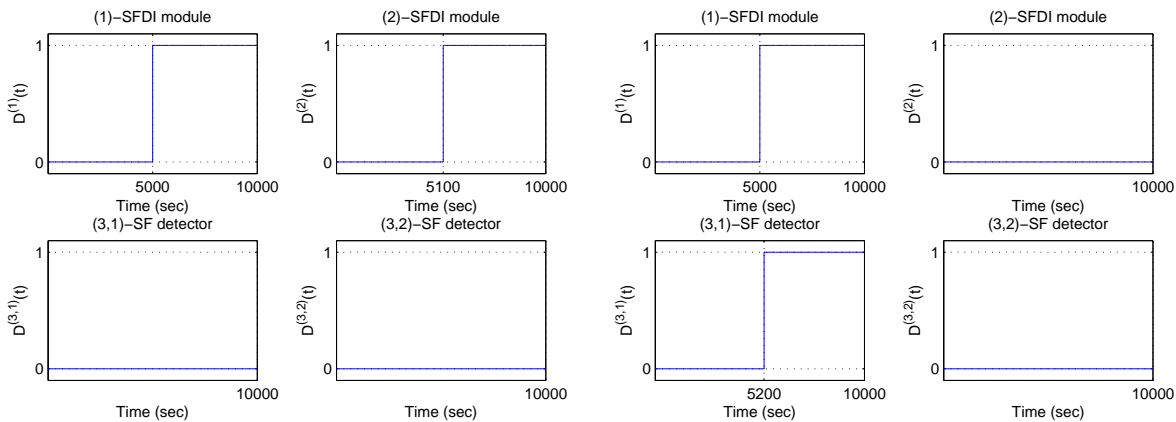

(a) Scenario 1
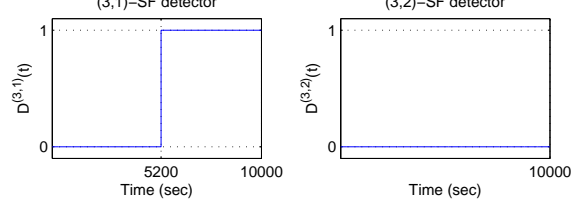

(b) Scenario 2
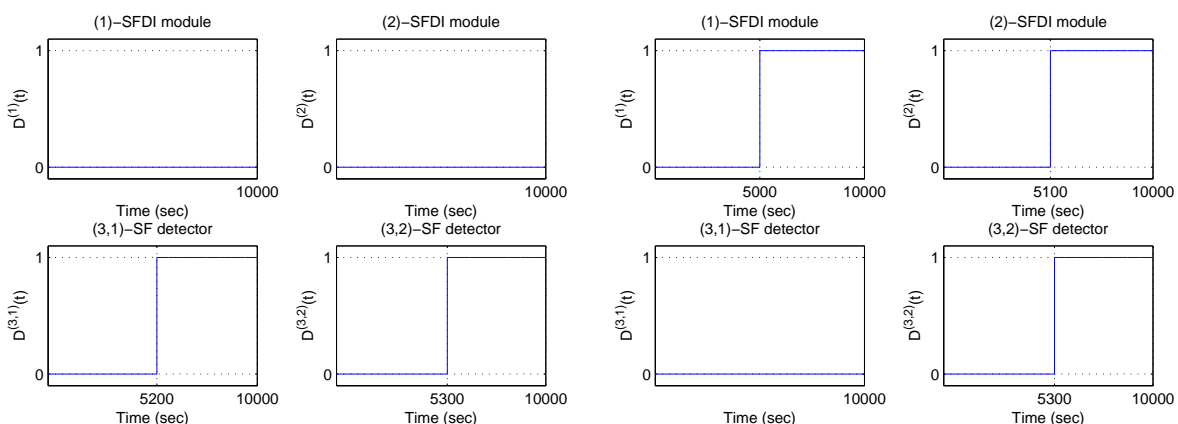

(c) Scenario 3

(d) Scenario 4

Fig. 5. Distributed sensor fault detection and isolation decision in the case of multiple sensor faults 
to the theoretical sensor fault patterns of SFSMs, shown in Tables I and II, all sensor faults can be isolated at the time instances indicated in each plot. For example, based on Fig. 4(a), we can infer at the time instant $5000 \mathrm{sec}$ that the sensor measuring the test cell temperature $T_{t c_{1}}$ is faulty; based on Fig. 5(b), we may infer that at the time instant 5000 sec, the sensor measuring the test cell temperature $T_{t c_{1}}$ is faulty, while at the time instant $5200 \mathrm{sec}$, a fault in the sensor measuring the temperature in the cooling coil $T_{a o}$ has also occurred; or based on Fig. 5(d), we can conclude that the sensors measuring the temperature of the two test cells and the sensor that measures the temperature of the chiller water tank are faulty.

\section{CONCLUSIONS}

In this paper, we presented a model-based, distributed architecture for multiple sensor fault detection and isolation (SFDI) in a HVAC system. By modeling the HVAC system as a set of three interconnected subsystems (two zones and the electromechanical part), we design distributed sensor fault detection and isolation modules dedicated to each of the interconnected subsystems. The inference mechanism in each SF detector is implemented using robust analytical redundancy relations (ARRs), formulated by structured residuals and adaptive thresholds. The information generated by each SFDI module is integrated and processed using a reasoning-based decision logic for achieving multiple sensor fault isolation. Simulation results showed the effectiveness of the proposed distributed SFDI methodology in isolating multiple sensor faults in the interconnected subsystems.

\section{REFERENCES}

[1] K. W. J. Wong, "Development of selection evaluation and system intelligence analytic models for the intelligent building control systems," Ph.D. dissertation, The Hong Kong Polytechnic University, 2007.

[2] J. E. Braun, "Intelligent building systems-past, present, and future," in American Control Conference, 2007. IEEE, 2007, pp. 4374-4381.

[3] Energy Efficiency Office, "Hong kong energy end-use data," Electrical and Mechanical Services Department (EMSD), The Government of the Hong Kong Special Administrative Region, Tech. Rep., 1999.

[4] R. Isermann, Fault-diagnosis systems: an introduction from fault detection to fault tolerance. Springer Verlag, 2006.

[5] J. Chen and R. Patton, Robust model-based fault diagnosis for dynamic systems. Kluwer Academic Publishers, 1999.

[6] J. Schein, S. T. Bushby, N. S. Castro, and J. M. House, "A rule-based fault detection method for air handling units," Energy and buildings, vol. 38, no. 12, pp. 1485-1492, 2006.

[7] B. T. Thumati, M. A. Feinstein, J. W. Fonda, A. Turnbull, F. J. Weaver, M. E. Calkins, and S. Jagannathan, "An online model-based fault diagnosis scheme for hvac systems," in IEEE International Conference on Control Applications (CCA), 2011. IEEE, 2011, pp. 70-75.

[8] J. Liang and R. Du, "Model-based fault detection and diagnosis of hvac systems using support vector machine method," International Journal of refrigeration, vol. 30, no. 6, pp. 1104-1114, 2007.

[9] D. A. Veronica, "Detecting cooling coil fouling automatically -Part 2: Results using a multilayer perceptron," HVAC\&R Research, vol. 16, no. 5, pp. 599-615, 2010.

[10] J. Hyvärinen and S. Kärki, Building optimization and fault diagnosis source book. Technical Research Centre of Finland, VTT Building Technology, 1996.

[11] E. Miller-Hooks and T. Krauthammer, "An intelligent evacuation, rescue and recovery concept," Fire technology, vol. 43, no. 2, pp. 107-122, 2007.
[12] J. Zhang, C. Mohan, P. Varshney, C. Isik, K. Mehrotra, S. Wang, Z. Gao, and R. Rajagopalan, "Intelligent control of building environmental systems for optimal evacuation planning," in Proceedings of International Conference on Indoor Air Quality Problems and Engineering Solutions, 2003.

[13] B. Fan, Z. Du, X. Jin, X. Yang, and Y. Guo, "A hybrid fdd strategy for local system of ahu based on artificial neural network and wavelet analysis," Building and Environment, vol. 45, no. 12, pp. 2698-2708, 2010.

[14] X. Xu, F. Xiao, and S. Wang, "Enhanced chiller sensor fault detection, diagnosis and estimation using wavelet analysis and principal component analysis methods," Applied Thermal Engineering, vol. 28, no. 2, pp. 226-237, 2008.

[15] Z. Du and X. Jin, "Detection and diagnosis for sensor fault in hvac systems," Energy conversion and management, vol. 48, no. 3, pp. 693$702,2007$.

[16] S. M. Namburu, M. S. Azam, J. Luo, K. Choi, and K. R. Pattipati, "Data-driven modeling, fault diagnosis and optimal sensor selection for hvac chillers," IEEE Transactions on Automation Science and Engineering, vol. 4, no. 3, pp. 469-473, 2007.

[17] S. Wang and J. Qin, "Sensor fault detection and validation of vav terminals in air conditioning systems," Energy conversion and management, vol. 46, no. 15, pp. 2482-2500, 2005.

[18] S. Wang, Q. Zhou, and F. Xiao, "A system-level fault detection and diagnosis strategy for hvac systems involving sensor faults," Energy and buildings, vol. 42, no. 4, pp. 477-490, 2010.

[19] S. Katipamula and M. R. Brambley, "Review article: Methods for fault detection, diagnostics, and prognostics for building systemsa review, part i," HVAC\&R Research, vol. 11, no. 1, pp. 3-25, 2005.

[20] M.-L. Chiang and L.-C. Fu, "Hybrid system based adaptive control for the nonlinear hvac system," in American Control Conference, 2006. IEEE, 2006, pp. 6-pp.

[21] M. Zaheer-Uddin, R. V. Patel, and S. A. Al-Assadi, "Design of decentralized robust controllers for multizone space heating systems," IEEE Transactions on Control Systems Technology, vol. 1, no. 4, pp. 246-261, 1993.

[22] M. Zaheer-Uddin, "Temperature control of multizone indoor spaces based on forecast and actual loads," Building and Environment, vol. 29, no. 4, pp. 485-493, 1994.

[23] A. Thosar, A. Patra, and S. Bhattacharyya, "Feedback linearization based control of a variable air volume air conditioning system for cooling applications," ISA transactions, vol. 47, no. 3, pp. 339-349, 2008.

[24] W.-Y. Lee, J. M. House, and N.-H. Kyong, "Subsystem level fault diagnosis of a building's air-handling unit using general regression neural networks," Applied Energy, vol. 77, no. 2, pp. 153-170, 2004.

[25] S. Wang and J.-B. Wang, "Law-based sensor fault diagnosis and validation for building air-conditioning systems," HVAC\&R Research, vol. 5, no. 4, pp. 353-380, 1999.

[26] _ - "Robust sensor fault diagnosis and validation in hvac systems," Transactions of the Institute of Measurement and Control, vol. 24, no. 3, pp. 231-262, 2002.

[27] R. Escobar, C. Astorga-Zaragoza, A. Téllez-Anguiano, D. JuárezRomero, J. Hernández, and G. Guerrero-Ramírez, "Sensor fault detection and isolation via high-gain observers: Application to a doublepipe heat exchanger," ISA transactions, vol. 50, no. 3, pp. 480-486, 2011.

[28] A. Talukdar and A. Patra, "Dynamic model-based fault tolerant control of variable air volume air conditioning system," HVAC\&R Research, vol. 16, no. 2, pp. 233-254, 2010.

[29] V. Reppa, M. Polycarpou, and C. G. Panayiotou, "Multiple sensor fault detection and isolation for large-scale interconnected nonlinear systems," in European Control Conference (ECC2012) (submitted), Zurich, Switzerland, 2012.

[30] — , "Distributed sensor fault detection and isolation for nonlinear uncertain systems," in 8th IFAC SAFEPROCESS, Mexico City, Mexico, 2012.

[31] W. Chen and J. Li, "Decentralized output-feedback neural control for systems with unknown interconnections," IEEE Transactions on Systems, Man, and Cybernetics, Part B: Cybernetics, vol. 38, no. 1, pp. 258-266, 2008.

[32] M. Blanke, M. Kinnaert, J. Lunze, and M. Staroswiecki, Diagnosis and fault-tolerant control. Springer Verlag, 2003. 